\title{
Transcendental Semigroup that has Simply Connected Fatou Components
}

\author{
Bishnu Hari Subedi \\ Central Department of Mathematics, Institute of Science and Technology, Tribhuvan University, \\ Nepal \\ Corresponding author: subedi_bh@cdmathtu.edu.np
}

Received: Oct 9, 2018

Revised: Nov 26, 2018

Accepted: $\operatorname{Dec} 2,2018$

\begin{abstract}
We prove that there exists non-trivial transcendental semigroup S such that the periodic or pre-periodic or wandering components of Fatou set $\mathrm{F}(\mathrm{S})$ have simply connected domain D.
\end{abstract}

Keywords: Fatou set, periodic component, wandering component, transcendental semigroup, Carleman set.

AMS (MOS) Subject Classification: 37F10, 30D05.

\section{Introduction}

We denote the complex plane by $\mathbb{C}$, the Riemann sphere by $\mathbb{C}_{\infty}$ and set of integers greater than zero by $\mathbb{N}$. We assume the function $\mathrm{f}: \mathbb{C} \rightarrow \mathbb{C}$ is transcendental entire function (TEF) unless otherwise stated. For any $\mathrm{n} \in \mathbb{N}, \mathrm{f}^{n}$ always denotes the $\mathrm{nth}$ iterates of $\mathrm{f}$. If $\mathrm{f}^{\mathrm{n}}(\mathrm{z})=\mathrm{z}$ for some smallest $\mathrm{n} \in \mathbb{N}$, then we say that $\mathrm{z}$ is periodic point of period $\mathrm{n}$. In particular, if $\mathrm{f}(\mathrm{z})=\mathrm{z}$, then $\mathrm{z}$ is a fixed point of $\mathrm{f}$. If $\left|\left(\mathrm{f}^{\mathrm{n}}(\mathrm{z})\right)^{\prime}\right|<1$, where ' represents complex differentiation of $\mathrm{f}^{\mathrm{n}}$ with respect to $\mathrm{z}$, then $\mathrm{z}$ is called attracting periodic point. A family $\mathcal{F}=\{\mathrm{f}: \mathrm{f}$ is meromorphic on some domain $\mathrm{X}$ of $\mathbb{C}_{\infty}$ forms normal family if every sequence $(\mathrm{f})_{\mathrm{I} \in \mathbb{N}}$ of functions contains a subsequence which converges uniformly to a finite limit or converges to $\infty$ on every compact subset $\mathrm{D}$ of $\mathrm{X}$. The Fatou set of $\mathrm{f}$ denoted by $\mathrm{F}(\mathrm{f})$ is the set of point's $\mathrm{z} \in \mathbb{C}$ such that sequence $\left(\mathrm{f}^{\mathrm{n}}\right)_{n \in \mathbb{N}}$ forms a normal family in some neighborhood of $\mathrm{z}$. A maximally connected subset of the Fatou set F(f) is called Fatou component. By definition, Fatou set is open and may or may not be empty. Fatou set is non-empty for every entire function with attracting periodic points. The complement of Fatou set is called Julia set and it is denoted by $\mathrm{J}(\mathrm{f})$. Classically, it is known that the sets $F(f)$ and $J(f)$ form a fundamental partition of complex plane $\mathbb{C}$.

Let $\mathrm{U} \subset \mathrm{F}(\mathrm{f})$ (a Fatou component) such that $\mathrm{f}^{\mathrm{n}}(\mathrm{U})$ for some $\mathrm{n} \in \mathbb{N}$, is contained in some component of $F(f)$, which is usually denoted by $U_{n}$. A Fatou component $U$ is called pre-periodic if there exist integers $\mathrm{n}>\mathrm{m} \geq 0$ such that $\mathrm{U}_{\mathrm{n}}=\mathrm{U}_{\mathrm{m}}$. In particular, if $\mathrm{U}_{\mathrm{n}}=\mathrm{U}_{0}=\mathrm{U}$ ( that is, $\mathrm{f}$ ${ }^{n}(U) \subset U$ ) for some smallest positive integer $n \geq 1$, then $U$ is called periodic Fatou component 
of period $\mathrm{n}$ and $\left\{\mathrm{U}_{0}, \mathrm{U}_{1} \ldots, \mathrm{U}_{\mathrm{n}-1}\right\}$ is called the periodic cycle. A component of Fatou set $\mathrm{F}(\mathrm{f})$ which is not pre-periodic is called wandering domain.

Let us denote the class of all transcendental entire maps on $\mathbb{C}$ by $\mathcal{E}$. Our particular interest is to study of the dynamics of this family $\mathcal{E}$. For a collection $\mathcal{F}=\left\{\mathrm{f}_{\alpha}\right\}_{\alpha \in \Delta}$ of such maps, let us define a set $S=\left\langle f_{\alpha}\right\rangle$

generated by all maps $\mathrm{f}_{\alpha . .} \Delta$ is an index set to which $\alpha$ belongs is finite or infinite. This set $\mathrm{S}$ forms a semigroup under the binary operation of functional composition. This semigroup $\mathrm{S}$ is known as transcendental semigroup generated by transcendental entire function $\mathrm{f}_{\alpha}$. for all $\alpha$. Note that each $\mathrm{f} \in \mathrm{S}$ is constructed through the composition of finite number of functions $f_{\alpha_{k}},(\mathrm{k}=1,2,3, \ldots, \mathrm{m})$.

$$
\text { That is, } \mathrm{f}=f_{\alpha_{1}} \circ f_{\alpha_{2}} \circ \cdots \circ f_{\alpha_{m}} \text {. }
$$

A semigroup generated by finitely many transcendental functions $f_{i},(i=1,2 \ldots n)$ is called finitely generated transcendental semigroup. We write $S=\left\langle\mathrm{f}_{1}, \mathrm{f}_{2} \ldots \mathrm{f}_{\mathrm{n}}\right\rangle$. If $\mathrm{S}$ is generated by only one transcendental entire function $\mathrm{f}$, then $\mathrm{S}$ is cyclic semigroup. We write $\mathrm{S}=\langle\mathrm{f}\rangle$. In this case, each $g \in S$ can be written as $g=f^{n}$, where $f^{n}$ is the nth iterates of $f$ with itself. Note that in our study of semigroup dynamics, we say $\mathrm{S}=\langle\mathrm{f}\rangle$ a trivial semigroup.

In classical complex dynamics, each of Fatou set and Julia set are defined in two different but equivalent ways. In first definition, Fatou set is defined as the set of normality of the iterates of given function and Julia set is defined as the complement of the Fatou set. The second definition of Fatou set is given as a largest completely invariant open set and Julia set is given as a smallest completely invariant close set. Based on above first definition (that is, on the Fatou-Julia theory of a complex analytic function), the Fatou set and Julia set are defined as follows.

Definition 1: Fatou set of a transcendental semigroup $S$ is defined by

$$
F(S)=\{z \in \mathbb{C}: S \text { is normal in a neighborhood of } z\}
$$

and the Julia set $J(S)$ of $S$ is the compliment of Fatou set $F(S)$. Any maximally connected subset $U$ of the Fatou set $F(S)$ is called Fatou component.

Note that pre-periodic, periodic and wandering components of Fatou set are defined as in classical cases. If $S=\langle f\rangle$, then $F(S), J(S)$ and $I(S)$ are respectively the Fatou set and Julia set in classical complex dynamics. In this situation we simply write: $F(f)$ and $J(f)$. From the definition 1 , it is clear that $\mathrm{F}(\mathrm{S})$ is the open set and therefore, it's complement $\mathrm{J}(\mathrm{S})$ is closed set. Indeed, these definitions generalize the definitions of Julia set and Fatou set of the iteration of single holomorphic map.

The fundamental contrast between classical complex dynamics and semigroup dynamics appears by different algebraic structure of corresponding semigroup. In fact, non-trivial semigroup (rational or transcendental) need not be, and most often will not be abelian. However, trivial semigroup is cyclic and therefore abelian. As we discussed before, classical complex dynamics is 
a dynamical study of trivial (cyclic) semigroup whereas semigroup dynamics is a dynamical study of non-trivial semigroup.

Note that for any holomorphic semigroup $\mathrm{S}$, we have

- $F(S) \subset F(f)$ for all $f \in S$ and hence $F(S) \subset \bigcap_{f \in S} F(f)$.

- $\mathrm{J}(\mathrm{f}) \subset \mathrm{J}(\mathrm{S})$ for all $\mathrm{f} \in \mathrm{S}$.

Since, in classical complex dynamics, Fatou set F(f) may be empty. So from the above first relation, we can say that Fatou set $F(S)$ of semigroup $S$ may also be empty.

In this paper we are interested to find a non-trivial semigroup $S$ that has non-empty Fatou set $\mathrm{F}(\mathrm{S})$. Basically, we prove that there is a non-trivial transcendental semigroup that has simply connected Fatou component.

Theorem 1: There is a non-trivial transcendental semigroup $S$ such that the Fatou set $F(S)$ has at least a simply connected component.

Note that if the semigroup $S$ is trivial, that is, semigroup $S=\langle f\rangle$ generated by a single TEF $f$, then Bergweiler [1] proved that the Fatou set $\mathrm{F}(\mathrm{S})$ has both a simply and a multiply connected wandering domains. However, in the case of non-trivial transcendental semigroup, the proof is not so easy. The reason behind is that the dynamics of individual TEFs differ largely from the dynamics of their composites.

\section{Auxiliary Results}

To work out a proof of above theorem 1, first of all we need a notion of approximation theory of entire functions. In our case, we can use the notion of Carleman set from which we obtain approximation of any holomorphic map by transcendental entire functions.

Definition 2: Let $F$ be a closed subset of $\mathbb{C}$ and $C(F)=\{\mathrm{f}: F \rightarrow \mathbb{C}: \mathrm{f}$ is continuous on $F$ and analytic in the interior $F^{\circ}$ of $\left.F\right\}$. Then $\mathrm{C}(\mathrm{F})$ is called a Carleman set (for $\mathbb{C}$ ) if for any $g \in C(F)$ and any positive continuous function $\epsilon$ on $F$, there exists entire function $h$ such that $\mid g(z)$ $h(z) \mid<\epsilon$ for all $z \in F$.

The following important characterization of Carleman set was proved by Nersesjan in 1971 but we have been taken this result from the work of Gaier [2].

Theorem 2: Let $F$ be proper subset of $\mathbb{C}$. Then $F$ is a Carleman set for $\mathbb{C}$ if and only if $F$ satisfies the following:

a. $\mathbb{C}_{\infty}-F$ is connected;

b. $\mathbb{C}_{\infty}-F$ is locally connected at $\infty$;

c. for every compact subset $K$ of $\mathbb{C}$, there is a neighborhood $V$ of $\infty$ in $\mathbb{C}_{\infty}$ such that no component of $F^{\circ}$ intersects both $K$ and $V$. 
It is well known in classical complex analysis that the space $\mathbb{C}_{\infty}-\mathrm{F}$ is connected if and only if each component $\mathrm{Z}$ of open set $\mathbb{C}-\mathrm{F}$ is unbounded. This fact together with theorem 2 will be a nice tool whether a set is a Carleman set for $\mathbb{C}$. The sets given in the following examples are Carleman sets for $\mathbb{C}$.

Example 1: The set $\mathrm{E}=\{\mathrm{z} \in \mathbb{C}:|\mathrm{z}|=1, \operatorname{Re} \mathrm{z}>0\} \cup\{\mathrm{z}=\mathrm{x}: \mathrm{x}>1\} \cup \bigcup_{n=3}^{\infty}\left\{z=r e^{i \theta}: r>\right.$ $1, \theta=\pi / n\}$ is a Carleman set for $\mathbb{C}$.

Example 2: The set $\mathrm{E}=\mathrm{G}_{0} \cup \cup_{n=1}^{\infty}\left(G_{k} \cup B_{k} \cup L_{k} \cup M_{k}\right)$, where

- $\mathrm{G}_{0}=\{\mathrm{z} \in \mathbb{C}:|\mathrm{z}-2| \leq 1\}$;

- $\mathrm{G}_{\mathrm{k}}=\{\mathrm{z} \in \mathbb{C}:|\mathrm{z}-(4 \mathrm{k}+2)| \leq 1\} \cup\{\mathrm{z} \in \mathbb{C}: \operatorname{Re} \mathrm{z}=4 \mathrm{k}+2, \operatorname{Im} \mathrm{z} \geq 1\} \cup\{\mathrm{z} \in \mathbb{C}: \operatorname{Re} \mathrm{z}=$ $4 \mathrm{k}+2, \operatorname{Im} \mathrm{z} \leq-1\},(\mathrm{k}=1,2,3, \ldots)$;

- $\mathrm{B}_{\mathrm{k}}=\{\mathrm{z} \in \mathbb{C}:|\mathrm{z}+(4 \mathrm{k}+2)| \leq 1\} \cup\{\mathrm{z} \in \mathbb{C}: \operatorname{Re} \mathrm{z}=-(4 \mathrm{k}+2), \operatorname{Im} \mathrm{z} \geq 1\} \cup\{\mathrm{z} \in \mathbb{C}: \operatorname{Re}$ $\mathrm{z}=-(4 \mathrm{k}+2), \operatorname{Im} \mathrm{z} \leq-1\},(\mathrm{k}=1,2,3, \ldots)$;

- $\mathrm{L}_{\mathrm{k}}=\{\mathrm{z} \in \mathbb{C}: \operatorname{Re} \mathrm{z}=4 \mathrm{k}\},(\mathrm{k}=1,2, \ldots)$;

- $\mathrm{M}_{\mathrm{k}}=\{\mathrm{z} \in \mathbb{C}: \operatorname{Re} \mathrm{z}=-4 \mathrm{k}\},(\mathrm{k}=1,2,3 \ldots)$.

Then, by theorem 2, we can easily show that $\mathrm{E}$ is a Carleman set for $\mathbb{C}$. From the help of the Carleman set of example 2, A.P. Singh [4] proved the following result.

Lemma 1: There are transcendental entire functions $f$ and $g$ such that there exists a domain which lies in the wandering component of the $F(\mathrm{f}), F(g), F(\mathrm{f} \circ g)$ and $F(g \circ f)$.

In fact, Singh [4] also proved other results regarding the dynamics of two individual functions and their composites (see for instance [Theorem 1, Theorem 3 and Theorem 4]) which are also strictly based on the Carleman set of example 2. Dinesh Kumar, Gopal Datt and Sanjay Kumar [3] extended these result of Singh (see for instance [Theorem 2.1 to Theorem 2.15] of [3]). For our purpose, we cite their following results.

Lemma 2: There are transcendental entire functions $f$ and $g$ such that there exist infinite number of domains which lie in the pre-periodic component of the $F(\mathrm{f}), F(g), F(\mathrm{f} \circ g)$ and $F(g \circ$ f).

Lemma 3: There are transcendental entire functions $f$ and $g$ such that there exist infinite number of domains which lie in the wandering component of the $F(\mathrm{f}), F(g), F(\mathrm{f} \circ g)$ and $F(g \circ \mathrm{f})$ We have extended above lemmas 1, 2 and 3 to the following results:

Lemma 4: There are transcendental entire functions $f, g$ and $h$ such that there exist infinite number of domains which lie in the periodic component of the $F(\mathrm{f}), F(g), F(h), F(\mathrm{f} \circ g), F(g \circ$ $\mathrm{f}), F(f \circ h), F(g \circ h), F(h \circ f), F(h \circ g), F(f \circ g \circ h), F(f \circ h \circ g), F(g \circ f \circ h), F(g \circ h \circ f)$, $F(h \circ f \circ g)$ and $F(h \circ g \circ f)$

Lemma 5: There are transcendental entire functions $f, g$ and $h$ such that there exist infinite number of domains which lie in the pre-periodic component of $F(\mathrm{f}), F(g), F(h), F(\mathrm{f} \circ g), F(g \circ$ 
f), $F(f \circ h), F(g \circ h), F(h \circ f), F(h \circ g), F(f \circ g \circ h), F(f \circ h \circ g), F(g \circ f \circ h), F(g \circ h \circ f), F(h \circ$ $\mathrm{f} \circ \mathrm{g})$ and $F(h \circ g \circ \mathrm{f})$.

Lemma 6: There are transcendental entire functions $f, g$ and $h$ such that there exist infinite number of domains which lie in the wandering component of the $F(\mathrm{f}), F(g), F(h), F(\mathrm{fo} g)$, $F(g \circ f), F(f \circ h), F(g \circ h), F(h \circ f), F(h \circ g), F(f \circ g \circ h), F(f \circ h \circ g), F(g \circ f \circ h), F(g \circ h \circ$ f), $F(h \circ f \circ g)$ and $F(h \circ g \circ f)$.

Note that we have proved above lemmas 4, 5 in our unpublished work (pre-print) Subedi and Singh $[6,7]$ and Lemma 6 is published in [4]

\section{Proof of the theorem 1}

From above three lemmas 4, 5 and 6, we can say that whatever domains that lie in the periodic, pre-periodic and wandering components of $F(f), F(g), F(h), F(f \circ g), F(g \circ f), F(f \circ h), F(g \circ h)$, $F(h \circ f), F(h \circ g), F(f \circ g \circ h), F(f \circ h \circ g), F(g \circ f \circ h), F(g \circ h \circ f), F(h \circ f \circ g)$ and $F(h \circ g \circ$ f), they also lie respectively in the periodic, pre-periodic and wandering components of their successive composites.

Theorem 3: If $\mathrm{D}$ is a set which lies in the pre-periodic (or periodic or wandering) component of $\mathrm{F}(\mathrm{f}), \mathrm{F}(\mathrm{g}), \mathrm{F}(\mathrm{f} \circ \mathrm{g})$ and $\mathrm{F}(\mathrm{g} \circ \mathrm{f})$, then it also lies in pre-periodic (or periodic or wandering) component of $\mathrm{F}\left(f^{n_{k}} \circ g^{n_{k-1}} \circ \ldots \circ g^{n_{1}}\right)$ and $\mathrm{F}\left(g^{n_{k}} \circ f^{n_{k-1}} \circ \ldots \circ f^{n_{1}}\right)$, where $\mathrm{n}_{\mathrm{k}}, \ldots, \mathrm{n}_{1} \in \mathbb{N}$.

Proof: By the lemmas 2 and 3, such a set D exists. Since F (f) $=F\left(f^{n}\right)$ and F $(g)=F\left(g^{n}\right)$ for any $n \in \mathbb{N}$. So $D$ lies in the pre-periodic (or periodic or wandering) component of $F\left(f^{n}\right)$ and $F\left(g^{n}\right)$ for all $n \in \mathbb{N}$. As $D$ lies in the pre-periodic (periodic or wandering) component of $F(f \circ g)$, it also lies in the pre-periodic (or periodic or wandering) component of $F\left(f^{n} \circ g^{n}\right)$ for all $n \in \mathbb{N}$. By the same argument we are using here, $\mathrm{D}$ also lies in the pre-periodic (or periodic or wandering) component of $F(f \circ g)^{n}$ for all $n \in \mathbb{N}$. Since $F(f \circ g)^{n}=F(f \circ g \circ \ldots \circ f \circ g)(n$-times $f \circ g), D$ lies in the pre-periodic (or periodic or wandering) component of $F\left(f^{n} \circ g^{n} \circ \ldots \circ f^{n} \circ g^{n}\right)(n-$ times $f^{n} \circ g^{n}$ ) for all $n \in \mathbb{N}$. Since $n \in \mathbb{N}$ is arbitrary, so we conclude that $D$ lies in the pre-

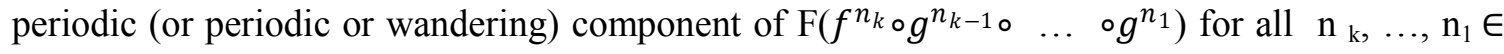
$\mathbb{N}$. Similarly, we can show that D lies in the pre-periodic (or periodic or wandering) component

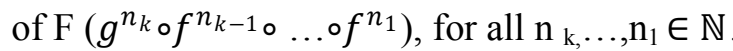

Theorem 4: If $\mathrm{D}$ is a set which lies in the periodic (or pre-periodic or wandering) component of $F(f), F(g), F(h), F(f \circ g), F(g \circ f), F(f \circ h), F(g \circ h), F(h \circ f), F(h \circ g), F(f \circ g \circ h), F(f \circ h \circ g)$, $\mathrm{F}(\mathrm{g} \circ \mathrm{f} \circ \mathrm{h}), \mathrm{F}(\mathrm{g} \circ \mathrm{h} \circ \mathrm{f}), \mathrm{F}(\mathrm{h} \circ \mathrm{f} \circ \mathrm{g})$ and $\mathrm{F}(\mathrm{h} \circ \mathrm{g} \circ \mathrm{f})$, then it also lies in the periodic (pre-periodic or wandering) component of $\mathrm{F}\left(f^{n_{k}} \circ g^{n_{k-1}} \circ h^{n_{k-2}} \ldots \circ f^{n_{1}}\right), \quad \mathrm{F}\left(g^{n_{k}} \circ f^{n_{k-1}} \circ h^{n_{k-2}} \ldots \circ g^{n_{1}}\right)$, and $\mathrm{F}\left(h^{n_{k}} \circ g^{n_{k-1}} \circ f^{n_{k-2}} \ldots \circ h^{n_{1}}\right)$, etc.

Proof: By lemmas 4, 5 and 6, such set D exists. By the similar argument of above theorem 1, the proof of this theorem follows. 
We prove the theorem 1 for a semigroup generated by two or three TEFs as defined in above lemmas $1,2,3,4,5$ and 6 .

Proof of the Theorem 1: Let $\mathrm{S}$ is a transcendental semigroup generated by two or three TEFs. If $\mathrm{S}$ is generated by two TEFs $\mathrm{f}$ and $\mathrm{g}$ as defined in the lemmas 2 and 3 , then by the theorem 3 , there is at least a domain which lies in the pre-periodic (or periodic or wandering) component of the $\mathrm{F}\left(f^{n_{k}} \circ g^{n_{k-1}} \circ \ldots \circ g^{n_{1}}\right)$ and $\mathrm{F}\left(g^{n_{k}} \circ f^{n_{k-1}} \circ \ldots \circ f^{n_{1}}\right)$, for all $\mathrm{n}_{\mathrm{k}}, \ldots, \mathrm{n}_{1} \in \mathbb{N}$. By the definition transcendental semigroup, any $\mathrm{h} \in \mathrm{S}=\langle\mathrm{f}, \mathrm{g}\rangle$ can be written in either of the form $\mathrm{h}$ $=f^{n_{k}} \circ g^{n_{k-1}} \circ \ldots \circ g^{n_{1}}$ or $\mathrm{h}=g^{n_{k}} \circ f^{n_{k-1}} \circ \ldots \circ f^{n_{1}}$, for all $n_{k}, \ldots, n_{1} \in \mathbb{N}$. Therefore, there is a domain D which lies in the pre-periodic (or periodic or wandering) component of the Fatou set $\mathrm{F}(\mathrm{h})$ for every function $\mathrm{h}$ of transcendental semigroup $\mathrm{S}$. This shows that this domain lies in the pre-periodic (or periodic or wandering) component of the Fatou set $F(S)$. Since for TEF, preperiodic (or periodic) domains are simply connected and so a domain within simply connected domains is also simply connected. The construction of the functions in the lemmas 1 and 3 , the domain which lies in the wandering domains is simply connected. If $\mathrm{S}$ is generated by three TEFs $\mathrm{f}, \mathrm{g}$ and $\mathrm{h}$ as defined in the lemmas 4, 5 and 6, then by theorem 4 and similar argument as above, Fatou set $\mathrm{F}(\mathrm{S})$ contains simply connected domain.

\section{Concluding Remarks}

Theorem 1 is our main result and it is a generalization of the results of Dinesh Kumar and Sanjay Kumar [3] but we restricted our proof of this theorem to the transcendental semigroup generated by three TEFs. Rigorously, it is not known that the essence of this theorem holds if a semigroup is generated by more than three TEFs and it is indeed an open problem of coming research. We can only say intuitively, the essence of this theorem 1 may hold if semigroup $\mathrm{S}$ is generated by $\mathrm{n}$ TEFs. There is another strong aspect of this theorem which is-if a transcendental semigroup generated by such type of three TEFs, then the Fatou set is non-empty.

\section{References}

[1] Bergweiler W (2011), Entire functions with simply and multiply connected wandering domains, Pure Appl. Math. Quarterly, 7: 107-120.

[2] Gaier A (1987), Lectures on complex approximation, Birkhauser.

[3] Kumar D and Kumar S (2015), Dynamics of composite entire functions, arXiv: 1207.5930v5 [math.DS].

[4] Singh AP (2003), On the dynamics of composite entire functions, Math. Proc. Camb. Phil. Soc. 134: 129-138.

[5] Subedi BH and Singh A (2018), Dynamics on the wandering components of Fatou set of three transcendental entire functions and their composites, arXiv: 1803.09259 v1 [math DS].

[6] Subedi BH and Singh A (2018), Dynamics on the periodic components of Fatou set of three transcendental entire functions and their composites, Preprint.

[7] Subedi BH and Singh A (2018), Dynamics on the pre-periodic components of Fatou set of three transcendental entire functions and their composites, Preprint. 